\title{
Survival of environmental DNA in natural environments and implications for the evolution of life.
}

\author{
L. Dieudonné, ${ }^{1,2}$ J. Q. SAnZ, ${ }^{1}$ S. Jelavić AND K.K. \\ SAND $* 1,3$
}

${ }^{1}$ GLOBE Institute, Section for GeoGenetics, University of Copenhagen, Denmark

${ }^{2}$ École nationale supérieure de chimie de Mulhouse, Université de Haute-Alsace, France

${ }^{3}$ Aberystwyth University, Geography \& Earth Sciences, United Kingdom

Minerals can prevent degradation of DNA and old DNA associated with minerals are now almost routinely used for e.g. genome reconstructions. DNA adsorbed to minerals can survive across timescales and be available to organisms that can incorporate the DNA into their genome through a process called horizontal gene transfer. Here we argue that minerals hold an unrecognised potential for transporting adaptive traits across environments and timescales to distant organisms impacting the evolution of life. ${ }^{1}$

The preservation potential and the availability of mineraladsorbed DNA for horizontal gene transfer should essentially depend on the mineral species. However, we have little insight into the underlying mechanisms that define and control DNA-mineral interactions and we cannot quantitatively address the stability of particular DNA-mineral bonds across timescales.

We have used atomic force microscopy (standard and video-rate imaging) to show that mineral surface charge, and the environmental conditions control the longevity of adsorbed DNA, and influence the conformation of the adsorbed DNA. We discuss which minerals and environmental conditions that could preserve DNA across time and space and which combinations are likley to promote mineral facilitated horizontal gene transfer and the potential for impacting the evolution of life.
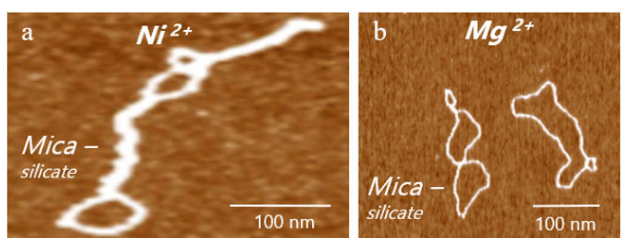

Plasmid DNA adsorbed to a negativley charged mineral surface. The DNA is a) supercoiled in $\mathrm{NiCl}_{2}$ b) circular and twisted in a $\mathrm{MgCl}_{2}$

1 Sand, K. K.; Jelavić, S. Mineral Facilitated Horizontal Gene Transfer: A New Principle for Evolution of Life? Frontiers in Microbiology 2018, 9. 